\title{
A REVIEW: DIFFERENTIAL TRANSFORM METHOD FOR SEMI- ANALYTICAL SOLUTION OF DIFFERENTIAL EQUATIONS
}

\author{
M.M. RASHIDI* \\ Shanghai Automotive Wind Tunnel Center, Tongji University \\ Shanghai 201804, CHINA \\ E-mail:mm_rashidi@yahoo.com; mm_rashidi@tongji.edu.cn \\ F. RABIEI and S. NASERI NIA \\ School of Engineering, Monash University Malaysia \\ 47500, Selangor, MALAYSIA \\ S. ABBASBANDY \\ Department of Applied Mathematics \\ Imam Khomeini International University \\ Qazvin, IRAN
}

\begin{abstract}
In this article, the semi-analytical method known as the Differential Transform Method (DTM) for solving different types of differential equations is reviewed. First, basic definitions and formulas of DTM and Differential Transform-Padé approximation (DTM-Padé), which are used to increase the convergence and accuracy of DTM approximations, are discussed. Then both techniques of DTM and DTM-Padé, which have been successfully applied to partial differential equations, as well as the application of these methods in fluid mechanic and heat transfer are presented. In addition, the extension of DTM for integral differential equations and the fuzzy differential transformation method (FDTM) for fuzzy problems are discussed.
\end{abstract}

Key words: differential transform method, differential transform-Pad'e approximation, semi-analytical method, differential equations, fuzzy differential transformation method, gS-differentiability.

\section{Introduction}

The Differential Transform Method (DTM) is an analytical and in some cases semi-analytical approach to solve differential equations. Initially, Zhou [28] proposed the idea of differential transform. He used the DTM to solve linear and nonlinear problems of an electric circuit. The DTM provides exact values of the $k$-th derivative of an analytical function at a point based on known and unknown initial boundary conditions. This method gives the analytical solution of differential equations in the form of a polynomial. It is different from the classical high order Taylor series method. The DTM is an iterative method to obtain the analytical Taylor series solutions of differential equations, while in the Taylor series method, symbolic computation of required derivatives of functions is necessary. That causes the high computational cost for high orders of the Taylor series method. In other words, the DTM gives the approximate solution of differential equations using the finite Taylor series, while there is no need to evaluate the derivatives of a function directly and an iteration procedure will be used to approximate the relative derivatives. In recent years, the DTM has been investigated and applied to solve various types of linear and nonlinear problems.

In the classic strategy, polynomials are useful for approximating the truncated power series. In addition, polynomials singularities cannot be found easily in a finite plane. Furthermore, using the power

\footnotetext{
* To whom correspondence should be addressed
} 
series is not always useful when the radius of convergence of the power series does not cover the two boundaries. Padé approximants is a useful tool to tackle this problem. The DTM-Padé uses rational functions of finite degree polynomials. Padé approximants are widely used in computational mathematics because a Padé approximant usually gives more accurate approximation of the function rather than truncating its power series. Padé approximants can also be used when the power series is not convergent. In addition, Padé approximants can be simply calculated by computer programming software such as Mathematica or Maple.

Fuzzy set theory is a powerful tool to model the uncertainty and process the mathematical model's data, which is used to for modelling of many real problems. Fuzzy differential equations are used as a natural technique for modelling dynamical systems with the presence of uncertainty [38]. In recent years, many research works and studies on theoretical and applied fields have been carried out and produced significant results in both areas, see $[10,18,19,32]$. There are various types of analytical and numerical techniques to solve the fuzzy initial value problem (FIVP). The initial studies in the field have usually pursued methods based on the Hukuhara derivative, which is one of common techniques that researchers used for solving FDEs. However, in some problems the Hukuhara derivative has poses difficulties that are considered as disadvantages of this approach. Therefore, in general due to the technique of fuzzification of used derivative of FDE formulas with the Hukuhara derivative, an efficient generalization of the related crisp case was not found. Moreover, the other well-known methods to solve FDEs are computationally expensive and they require high computational evaluations.Bede and Gal [7-9] introduceda more general definition of differentiable fuzzy mappings based on generalized differentiability to solve the disadvantages of the Hukuhara derivative. For first time, Allahviranloo et al. in [1], developed the DTM using the concept of generalized differentiability and introduced thefuzzy differential transformation method to solve first order fuzzy differential equation.

In this review paper, first, a brief introduction of DTM is given, and then a review of some applications of the DTM in fluid mechanic and heat transfer are discussed. Then, the DTM for integral differential equations and fuzzy differential equations are reviewed.

\section{Differential Transform Method (DTM)}

Consider $f(t)$ a single variable function; transformation of $k$-th derivative of $f(t)$ is defined by

$$
F(k)=\frac{1}{k !}\left[\frac{d^{k} f(t)}{d t^{k}}\right]_{t=t_{0}}
$$

where $F(K)$ is known as a transformed function. A differential inverse transform of $F(K)$ is given by

$$
\begin{aligned}
& f(t)=\sum_{k=0}^{\infty} F(k)\left(t-t_{0}\right)^{k}, \\
& f(t) \approx \sum_{k=0}^{i} F(k)\left(t-t_{0}\right)^{k} .
\end{aligned}
$$

In fact, from Eq.(1.1) and Eq.(1.3), we obtain

$$
F(k) \approx \sum_{k=0}^{i}\left[\frac{d^{k} f(t)}{d t^{k}}\right]_{t=t_{0}} \frac{\left(t-t_{0}\right)^{k}}{k !} .
$$


From Eqs (2.1) and (2.3), the mathematical formulas of the DTM are obtained and shown in Tab.2.

Table 2. Differential transform formulas.

\begin{tabular}{|l|l|}
\hline$f(t)=g(t) \pm h(t)$ & $F(k)=G(k) \pm H(k)$ \\
\hline$f(t)=c g(t)$ & $F(k)=c G(k), \quad c$ is constant \\
\hline$f(t)=\frac{d^{n} g(t)}{d t^{n}}$ & $F(k)=\frac{(k+n) !}{k !} G(k+n)=\sum_{m=0}^{k} G(m) H(k-m)$ \\
\hline$f(t)=g(t) h(t)$ & $F(k)=\delta(k-m), \quad \delta(k-m)= \begin{cases}1 & k=m \\
0 & k \neq m\end{cases}$ \\
\hline$f(t)=t^{n}$ & $F(k)=\frac{G(k-1)}{k}, \quad$ where $k>1$ \\
\hline$f(t)=\int_{0}^{t} g(y) d y$ & $F(k)=\sum_{s=0}^{k} \sum_{m=0}^{k-s} G(s) H(m) W(k-s-m)$ \\
\hline$f(t)=g(t) h(t) w(t)$ & \\
\hline
\end{tabular}

\section{Differential transform-Padé technique}

The technique of differential transform-Padé approximation can increase the accuracy of series solution given in Eq.(2.4) with a higher rate of convergence, when compared with DTM solutions [42].

Summation theory is used to obtain the convergent function $f(t)$ while Euler summation evaluates the limit of the convergent series, and in Borel, summation gives the limit of a convergent integral. In Euler and Borel summation, the exact value of all of divergent series terms must be known in advance, while in real calculation only a limited number of series terms can be evaluated. Therefore, it is essential to have a summation algorithm to input only a finite number of divergent series terms. Then, for given new terms, a new and improved approximate of an exact sum of the divergent series is available. One of the best summation methods with this advantage is Padé approximation.

Liao in [21] proposed the Padé approximation as a powerful tool to increase and improve the accuracy and convergence of the series. The main idea of Padé summation is to change and substitute the value of

$$
f(t)=\sum_{i=0}^{+\infty} c_{i} t^{i}
$$

with a sequence of a rational function of two polynomials given by

$$
f_{q}^{p}(t)=\frac{\sum_{i=0}^{p} a_{i} t^{i}}{\sum_{j=0}^{q} b_{j} t^{j}}
$$

where with no loss of generality we consider $b_{0}=1$. Then choose other $(p+q+1)$ values of coefficients $a_{0}, a_{1}, \ldots, a_{p} ; b_{1}, \ldots, b_{q}$, so that the first $(p+q+1)$ the terms in the power series expansion of $f_{q}^{p}(t)$ are 
same as first $(p+q+1)$ terms of the power series $f(t)=\sum_{i=0}^{+\infty} c_{i} t^{i}$. The obtained rational function $f_{q}^{p}(t)$ is called a Padé approximate. If the power series $\sum_{i=0}^{+\infty} c_{i} t^{i}$ represents the function $f(t)$, then in many cases $f_{q}^{p}(t) \rightarrow f(t)$ as $\quad p, q \rightarrow \infty$, even when the series $\sum_{i=0}^{+\infty} c_{i} t^{i}$ diverges. The convergence of the Padé sequences given by

$$
f_{0}^{l}+f_{1}^{1+l}+f_{2}^{2+l}
$$

will usually be considered, where $p=q+l$ with a fixed value of land $q \rightarrow \infty$. When $l=0$ then this sequence is a known as a diagonal sequence.

The differential transform is combined with Padé technique and called the differential transformPadé approximation, which is found to be a very efficient tool for solving differential equations.

\section{DTM in fluid mechanics and heat transfer}

Rashidi and Erfani [28] compared the numerical solution of Burgers' equation and nonlinear heat transfer problems by different models: the differential transform method and the homotopy analysis method. Keimanesh et al. [20] used the multi-step differential transform method to study a non-Newtonian fluid flow with third grade and two parallel plates. Meanwhile, Mohyud-Din et al. [23] applied the DTM for obtaining a semi-analytic solution of heat transfer analysis for the squeezing flow of a Casson fluid with parallel circular plates. By using the similarity transformation, the model has been reduced to an equivalent system of nonlinear ODEs.

In [36], the DTM is used to approximate the heat transfer and unsteady two phases of a nanofluid flow under the influence of the magnetic field between moving parallel plates. The similarity transformation was applied to convert the partial differential equations into the system of ODEs. The DTM is specified as applicable for solving these problems, compared to solving with the fourth order Runge-Kutta method (RK4).

Rashidi [31] developed the DTM-Padé to solve the magnetohydrodynamic equations with boundarylayer. It was indicated that DTM solutions are solely verified for small values of independent variables, which is actually a usual behavior for most of the analytical methods such as the VIM, HPM and ADM. That means the valid solution only exist for independent variables in the range $[0,1]$, and for higher values of independent variables, one must implement another technique for tackling the problem. One solution is using the Padé approximant for increasing the convergence domain.

In [34], authors used the DTM for finding analytical solutions of governing equations of a nonNewtonian fluid flow using an axisymmetric channel and porous wall on turbine disk. The approach was adopted for a cooling application. The obtained results were compared with the numerical solution of RK4. They showed that the method is very efficient for solving a large system of non-linear differential equations. The analytical solution of a nanofluid hydrothermal flow with the presence of a variable magnetic field, using the DTM, was studied by Sheikholeslami and Ganji [33].

\section{DTM in differential equations}

Gökdoğan et al. [15] adopted a fast and effective algorithm for the multi-step differential transformation method (MsDTM) by improving the convergences of the DTM series. MsDTM has been successful in application for analytical solutions of some well know differential equations such as van der 
Pol, Quadratic Riccati, Duffing, and Rayleigh equations. The obtained results proved that theMsDTM performed efficiently for its time stepping and time processing.

The concept of 3D differential transform method and basic theorems were proposed by Ayaz [5] for the first time. The application of the 2D and 3D differential transform was investigated for exact solutions of both linear and non-linear systems of PDEs. In addition, Ayaz [4] proposed the numerical approximation of linear differential-algebraic equations (DAEs) by means of the DTM. Using this technique, different problems have been solved and numerical results were compared with exact solutions. The DTM can simply be applied to DAEs and series solutions. Ebrahimi et al. [12] investigated the applicability of the DTM on vibrational characteristics of functionally graded (FG) size-dependent nano-beams.

\section{DTM in integral -differential equations}

In [3], Arikoglu and Ozkol extended the DTM for solving integro-differential equations. New theorems are introduced and proved for the transformation of integrals. There are many linear and nonlinear integro-differential problems that were solved to demonstrate the efficiency of the method. Odibat [25] proposed the DTM for solving linear and nonlinear Volterra integral equations where the kernels were separable. The numerical solution of these problems is approximated in series form. The power and robustness of the DTM for solving complicated problems are studied in [2, 26, 27, 30, 35, 37].

\section{DTM in fuzzy differential equations}

In recent years, many researchers have studied the fuzzy differential transformation method (FDTM) for solving fuzzy differential equations. In [1] Allahviranloo et al., using the generalized differentiability concept, proposed FDTM for solving first order fuzzy differential equations. The fuzzy transformation $F(k)$ of the $k$-th derivative of the fuzzy function $f(t)$ in one variable is defined as follows

$$
F_{1}(k, r)=\frac{1}{k !}\left[\frac{d^{k} f_{1}(t)}{d t^{k}}\right]_{t=t_{0}}, \quad F_{2}(k, r)=\frac{1}{k !}\left[\frac{d^{k} f_{2}(t)}{d t^{k}}\right]_{t=t_{0}} \quad \forall k \in\{0,12, \ldots\}
$$

when $f(t)$ is the generalized Seikkala derivative (gS-derivative) in the form of (i) and

$$
\begin{aligned}
& F_{1}(k, r)=\frac{1}{k !}\left[\frac{d^{k} f_{2}(t)}{d t^{k}}\right]_{t=t_{0}}, \quad F_{2}(k, r)=\frac{1}{k !}\left[\frac{d^{k} f_{1}(t)}{d t^{k}}\right]_{t=t_{0}} \quad \forall k \text { is odd }, \\
& F_{l}(k, r)=\frac{1}{k !}\left[\frac{d^{k} f_{l}(t)}{d t^{k}}\right]_{t=t_{0}}, \quad F_{2}(k, r)=\frac{1}{k !}\left[\frac{d^{k} f_{2}(t)}{d t^{k}}\right]_{t=t_{0}} \forall k \text { is even },
\end{aligned}
$$

when $f(t)$ is gS-differentiable in the form of (ii).

$f(t)$ is the original fuzzy function, $F_{l}(k, r)$ and $F_{2}(k, r)$ are the transformed functions. The differential inverse transform is given as follows

$$
f_{l}(t)=\sum_{k=0}^{\infty} F_{1}(k, r)\left(t-t_{0}\right)^{k}, \quad f_{2}(t)=\sum_{k=0}^{\infty} F_{2}(k, r)\left(t-t_{0}\right)^{k},
$$


when $f(t)$ is gS-differentiable in the form of (i) and

$$
\begin{array}{ll}
f_{1}(t)=\sum_{k=0, \text { even }}^{\infty} F_{1}(k, r)\left(t-t_{0}\right)^{k}, & f_{2}(t)=\sum_{k=0, \text { odd }}^{\infty} F_{2}(k, r)\left(t-t_{0}\right)^{k}, \\
f_{l}(t)=\sum_{k=0, \text { even }}^{\infty} F_{2}(k, r)\left(t-t_{0}\right)^{k}, & f_{2}(t)=\sum_{k=0, \text { odd }}^{\infty} F_{l}(k, r)\left(t-t_{0}\right)^{k},
\end{array}
$$

where $f(t)$ is gS-differentiable in the form of (ii).

In [22], Mikaeilvand and Khakrangin used a two-dimensional FDTM to solve fuzzy partial differential equations. In [31], Salahshour and Allahviranloo used a new application of the FDTM for solving fuzzy Volterra integral equations, where the kernel was separable. In [6], Ahmadi and Kiani used the FDTM for solving fuzzy differential inclusions by fuzzy partitions. Ghazanfari and Ebrahimi used the FDTM for solving fuzzy fractional heat equations in [13]. They developed the generalized two-dimensional FDTM for the numerical solution of linear PDEs with space and time-fractional derivatives. Hajilou et al. considered the numerical solution of hybrid fuzzy differential equations by the FDTM in [16]. Mohammed and Khaleel used the FDTM for solving fuzzy integro-differential equations of fractional order in the Riemann-Liouville and Caputo fractional derivatives in [24]. The FDTM was proposed to approximate the solution of initial value for nonlinear fuzzy differential problems by Kadkhoda et al. in [17]. Ghazanfari and Ebrahimi used the FDTM for solving hybrid fuzzy differential equations in [14]. Biswas and Roy considered generalization of the Seikkala derivative and FDTM for fuzzy Volterra integro-differential equations in [11].

\section{Nomenclature}

DTM - Differential Transform Method

DTM-Padé - Differential Transform-Padé approximation

FDTM - Fuzzy differential transformation method

ODEs - Ordinary differential equations

PDEs - Partial differential equations

FDEs - Fuzzy differential equations

RK4 $-4^{\text {th }}$ order Runge-Kutta method

FIVP - Fuzzy initial value problem

\section{References}

[1] Allahviranloo T., Kiani N.A. and Motamedi N. (2009): Solving fuzzy differential equations by differential transformation method.-Information Sciences, vol.179, No.7, pp.956-966.

[2] Anwar Bég O., Tasveer A. Bég, Rashidi M.M. and Asadi M. (2013): DTM- Padé semi-numerical simulation of nanofluid transport in porous media.- International Journal of Applied Mathematics and Mechanics, vol.9, No.1, pp.80-107.

[3] Arikoglu A. and OzkolI (2005): Solution of boundary value problems for integro-differential equations by using differential transform method.- Appl. Math. Comput., vol.168,pp.1145-1158.

[4] Ayaz F. (2004): Application of differential transform method to differential-algebraic equations.- Appl. Math. Comput., vol.152, pp.649-657.

[5] Ayaz F. (2004): Solutions of the system of differential equations by differential transform method.- Appl. Math. Comput., vol.147, pp.547-567.

[6] Barkhordari Ahmadi M. and Kiani N.A. (2013): Differential transformation method for solving fuzzy differential inclusions by fuzzy partitions.- Int. J. Industrial Mathematics, vol.5, No.3, pp.237-249. 
[7] Bede B. and Gal S.G. (2004): Almost periodic fuzzy-number valued functions. -Fuzzy Sets and Systems, vol.147, pp.385-403.

[8] Bede B. and Gal S.G. (2005): Generalizations of differentiability of fuzzy number valued function with application to fuzzy differential equations.- Fuzzy Sets and Systems, vol.151, pp.581-599.

[9] Bede B., Imre J., Rudas C. and Attila L. (2007): First order linear fuzzy differential equations under generalized differentiability.-Information Sciences, vol.177, pp.3627-3635.

[10] Bencsik A., Bede B, Tar J. and Fodor J. (2006): Fuzzy Differential Equations in modeling hydraulic differential servo cylinders.- In: Third Romanian-Hungarian Join Symposium on Applied Computational Intelligence (SACI), Timisoara, Romania.

[11] Biswas S. and Roy T.K. (2018): Generalization of Seikkala derivative and differential transform method for fuzzy Volterra integro-differential equations. - Journal of Intelligent and Fuzzy Systems, vol.34, No.4, pp.2795-2806.

[12] Ebrahimi F., Ghadiri M., Salari E., Hoseini S. and Shaghaghi G. (2015):Application of the differential transformation method for nonlocal vibration analysis of functionally graded nanobeams.- Journal of Mechanical Science and Technology, vol.29, No.3, pp.1207-1215.

[13] Ghazanfari B. and Ebrahimi P. (2015): Differential transformation method for solving fuzzy fractional heat equations. - Int. J. Mathematical Modelling and Computations, vol.5, No.1, pp.81-89.

[14] Ghazanfari B. and Ebrahimi P. (2016): Differential transformation method for solving hybrid fuzzy differential equations.-Journal of Hyperstructures, vol.5, No.1, pp.69-83.

[15] Gökdoğan A., Merdan M. and Yildirim A. (2012): Adaptive multi-Adaptive multi-step differential transformation method to solving nonlinear differential equations.-Mathematical and Computer Modelling, vol.55, No.3-4, pp.761-769.

[16] Hajilou E., Paripour M. and Heidari H. (2015):Application of differential transform method to solve hybrid fuzzy differential equations.- Int. J. Mathematical Modelling and Computations, vol.5, No.3, pp.203-217.

[17] Kadkhoda N., Sadeghi Roushan S. and Jafari H. (2018): Differential transform method: A tool for solving fuzzy differential equations.- Int. J. Applied and Computational Mathematics, 4:33.

[18] Kaleva O. (1987): Fuzzy differential equations.- Fuzzy Sets and Systems, vol.24, pp.301-317.

[19] Kaleva O. (1990): The Cauchy problem for fuzzy differential equations.- Fuzzy Sets and Systems, vol.35, pp.389396.

[20] Keimanesh M., Rashidi M.M., Ali J. Chamkha and Jafaric R. (2011): Study of a third grade non-Newtonian fluid flow between two parallel plates using the multi-step differential transform method.- Computers and Mathematics with Applications, vol.62, No.8, pp.2871-2891.

[21] Liao S.J. (2003): Beyond perturbation: introduction to the homotopy analysis method. - CRC Press, Chapman Hall, Boca Raton.

[22] Mikaeilvand N. and Khakrangin S. (2012): Solving fuzzy partial differential equations by fuzzy two-dimensional differential transform method.- Neural Computing and Applications, vol.21, No.1, pp.307-312.

[23] Mohyud-Din, Usman Wei M. and Hamid W.M. (2018): A study of heat transfer analysis for squeezing flow of a Casson fluid via differential transform method.- Neural Computing and Applications, vol.30, No.10, pp.32533264.

[24] Mohammed O.H. and Khaleel O.I. (2016): Fractional differential transform method for solving fuzzy integrodifferential equations of fractional order. -Basrah Journal of Science (A), vol.4, No.2, pp.31-40.

[25] Odibat Z. (2008): The differential transform method for solving Volterra integral equation with separable kernels.- Mathematical and Computer Modelling, vol.48, No.7-8, pp.1144-1149.

[26] Paripour M., Karimi L. and Abbasbandy S. (2017): Differential transform method for Volterra's population growth model.- Nonlinear Studies, vol.42, No.1, pp.227-234. 
[27] Rashidi M.M. and Erfani E. (2009): A Novel Analytical Solution of the Thermal Boundary-Layer over a Flat Plate with a Convective Surface Boundary Condition Using DTM-Padé.- International Conference on Applied Physics and Mathematics (ICAPM 2009) Singapore.

[28] Rashidi M.M. and Erfani E. (2009): New analytical method for solving Burgers' and nonlinear heat transfer equations and comparison with HAM.- Computer Physics Communications, vol.180, pp.1539-1544.

[29] Rashidi M.M., Hayat T., Keimanesh T. and Yousefian H. (2013): A study on heat transfer in a second grade fluid through a porous medium with the modified differential transform method.- Heat Transfer-Asian Research, vol.42, No.1, pp.31-45.

[30] Rashidi M.M. (2009): The modified differential transform method for solving MHD boundary-layer equations.Computer Physics Communications, vol.180, No.11, pp.2210-2217.

[31] Salahshour S. and Allahviranloo T. (2013): Application of fuzzy differential transform method for solving fuzzy Volterra integral equations.- Applied Mathematical Modelling, vol.37, No.3, pp.1016-1027.

[32] Seikkala S. (1987): On the fuzzy initial value problem.- Fuzzy Sets and Systems, vol.24, pp.319-330.

[33] Sheikholeslami M. and Ganji D.D. (2014): Three dimensional heat and mass transfer in a rotating system using nanofluid.- Powder Technol., vol.253, pp.789-796, http://dx.doi.org/10.1016/j.powtec.2013.12.042

[34] Sepasgozar S., Faraji M. and Valipour P. (2017): Application of differential transformation method (DTM) for heat and mass transfer in a porous channel.- Propulsion and Power Research, vol.6, No.1, pp.41-48.

[35] Shukla1H.S., Tamsi M., Srivastava V.K. and Rashidi M.M. (2016):Modified cubic B-spline differential quadrature method for numerical solution of three dimensional coupled viscous burger equation.- Modern Physics Letters B, vol.30, No.11.

[36] Usman M., Hamida M., Khanb U., Mohyud Dinc S., Iqbald M.A. and Wei Wang (2018): Differential transform method for unsteady nanofluid flow and heat transfer.- Alexandria Engineering Journal, vol.57, No.3, pp.18671875.

[37] Yousif M., Mahmood B. and Rashidi M.M (2017): Using differential transform method and Padé approximant for solving MHD three-dimensional Casson fluid flow past a porous linearly stretching sheet.- Journal of Mathematics and Computer Science, vol.17, pp.169-178.

[38] Zadeh L. (2005): Toward a generalized theory of uncertainty (GTU) an outline.- Information Sciences, vol.172, 140.

[39] Zhou J.K. (1986): Differential transformation and its applications for electrical circuits.- Huazhong University Press, Wuhan, China.

[40] Zou L., Zong Z., Wang Z. and Tian S. ():Differential transform method for the degasperis-procesi equation. Advances in Electric and Electronics, LNEE, vol.155, pp.197-203.

Received: July 10, 2019

Revised: December 31, 2019 